\title{
El proceso intencionalldecisorio migratorio desde una aproximación cualitativa longitudinal
}

\author{
The intention/decision-making migration process from a \\ qualitative longitudinal approach
}

\author{
Renáta Hosnedlová \\ ATER Sciences Po Toulouse \\ Laboratoire d'Etude et de Recherche sur l'Economie, \\ les Politiques et les Systèmes Sociaux \\ renata.hosnedlova@gmail.com (FRANCIA)
}

Recibido: 01.112018

Aceptado: 30.01 .2020

\section{RESUMEN}

La perspectiva longitudinal cualitativa, aunque potencialmente adecuada, no ha recibido mucha atención en el campo de la migración internacional. Especialmente, las intenciones y decisiones residenciales de los migrantes se suelen estudiar desde un punto de vista estático, lo cual no permite explicar el fenómeno como proceso. El presente artículo demuestra la necesidad de una aproximación dinámica y cualitativa para explicar cómo se forman, mantienen o reformulan las intenciones / decisiones a lo largo del proyecto migratorio. Para ello, se utiliza un estudio de caso ucraniano en la Comunidad de Madrid, realizado en 4 oleadas entre los años 2009 y 2016. Mediante este estudio que articula las dimensiones temporales, espaciales y relacionales a la vez, se explican las aportaciones, pero también las dificultades y los retos conceptuales, metodológicos y analíticos de esta aproximación. Entre otros, se destaca la capacidad de observar los procesos causales cognitivos, lo que posibilita una mayor profundización sobre cómo se modelan las intenciones y toman decisiones residenciales.

\section{PALABRAS CLAVE}

Investigación longitudinal cualitativa, formación de la intención, toma de decisión, migración internacional, Ucranianos. 


\begin{abstract}
The qualitative longitudinal approach, although potentially adequate, has not received much attention in the field of international migration. Especially, the residential intentions and decisions of migrants are usually studied from a static point of view, which does not explain the phenomenon as a process. This article demonstrates the need for a dynamic and qualitative perspective to explain how intentions / decisions are formed, maintained or reformulated throughout the migratory project. For this, we use the Ukrainian case study in the Community of Madrid, carried out in 4 waves, between 2009 and 2016. Through this study that hat articulates the temporal, spatial and relational dimensions at the same time; we discuss the conceptual, methodological and analytical contributions and challenge of this approach. Among others, we highlight the ability to observe the cognitive causal processes, which allows a deeper understanding of how intentions are modelled and residential decisions are made.
\end{abstract}

\title{
KEY WORDS
}

Longitudinal qualitative research, intention formation, decision-making, international migration, Ukrainians.

\section{INTRODUCCIÓN: LA NECESIDAD DE UNA APROXIMACIÓN CUALITATIVA Y DINÁMICA A LAS INTENCIONES/DECISIONES MIGRATORIAS}

El interés en las decisiones migratorias tiene una larga historia en el campo de la migración internacional (p. ej. De Jong y Gardner 1981). Hasta el día de hoy se han desarrollado numerosos modelos y teorías para explicar por qué los individuos deciden emigrar de su país de origen a un país de destino (p. ej. Khoo 2003, Güngör y Tansel 2010). Un menor número de académicos se ha centrado en la decisión de retronar al país de origen, re-emigrar a otro país y/o participar a la migración circular (p. ej. Chavez 1994). Una minoría aborda la decisión migratoria como un proceso, desde su fase pre-decisoria (intencional) hasta la fase de la acción, y explorando la relación entre la fase intencional y la acción correspondiente. La revisión de la literatura científica evidencia que tanto las intenciones como las decisiones de (no)cambiar de residencia se han estudiado casi con exclusividad desde una perspectiva estática, preguntando sobre las intenciones en un momento específico y desvinculando la acción de la intención y de la fase preparatoria. Así, una gran parte de trabajos académicos intentan averiguar quién es más propenso de tener una u otra intención, pero no se centran en el proceso mismo, es decir, en cómo el sujeto llega a tomar la decisión. Los seguidores de la perspectiva estática analizan las intenciones residenciales en función de diversas variables individuales y/o contextuales, y pretenden ave- 
riguar cuál de ellas o qué conjunto de factores son determinantes. La mayoría de estos trabajos son de índole cuantitativa. A pesar de que este enfoque estático se ha revelado útil y adecuado para identificar algunos de los factores que influyen en la intención residencial, no es suficiente para explicar su formación. Aunque los autores intentan incluir en sus trabajos explicaciones de índole dinámica, en general, se trata de suposiciones no contrastadas, y se echan en falta interpretaciones basadas en una exploración más profunda sobre la fase pre-decisional. En realidad, las intenciones no ocurren así sin más, sino que se forman, mantienen o reformulan a lo largo del proceso migratorio. Como afirman Coulter et al. (2010) la intención se debería entender como una serie de procesos decisorios ocurridos durante un período de tiempo que constituyen fase pre-decisional. Por otro lado, Kley (2011) sugiere que el proceso decisorio que incluye la intención y el comportamiento migratorio podrían ser vistos como un ejemplo de causación acumulativa de la acción social. Estos dos estudios muestran que la cuestión temporal es crucial para la comprensión del fenómeno, pero su correcta introducción en el análisis conlleva ciertas complejidades que se deben, entre otras, a las siguientes razones: La primera, es que los sujetos son in/migrantes que llevan diferente tiempo en el destino, por lo que sus proyectos migratorios están en distintas fases de desarrollo, con diferentes grados de adaptación a la sociedad de acogida y con diferentes vivencias migratorias. Además, estos sujetos se pueden encontrar en diferentes fases del ciclo vital y, probablemente, no todos los elementos que influyen en el proceso decisorio tendrán el mismo peso para un determinado actor en diferentes momentos vitales. Como considera Kley (2011), los actores persiguen ciertos objetivos y metas dependiendo de sus situaciones biográficas, y las transiciones del curso de vida probablemente afectarán a estas preferencias. La segunda causa está relacionada con la variabilidad de la intención en el tiempo. Como ya ha sido demostrado en algunos estudios sobre fecundidad ${ }^{1}$ (Mitchell y Gray 2007), las intenciones no son estables, sino que evolucionan en el tiempo. Los proyectos vitales pueden ser modificados dependiendo, bien de las circunstancias externas que se presentan (ej. pérdida o cambio de trabajo, adquisición o expiración del permiso de residencia, cambios económicos y políticos de los países en cuestión, etc.), o bien por los cambios internos del sujeto (cambios de actitudes, cambios en estrategias, etc.), ya que la experiencia de la vida cotidiana en el extranjero deja huella en los propios sujetos y puede hacer que reconsideren sus intenciones. Asimismo, se ha examinado y demostrado en el estudio de Fliegenschnee (2006) que la decisión final sobre la acción intencionada se suele posponer en el caso de que las circunstancias actuales y la idea sobre las condiciones ideales no concuerden. La tercera causa es que la información de la que dispone el in/migrante para poder evaluar los riesgos y beneficios de sus futuras acciones varía en el tiempo y en función de la situación socio-económica del individuo y/o de su familia.

${ }^{1}$ Dado que sobre el proceso intencional / decisorio en migraciones existe relativamente poco conocimiento, nos hemos apoyado parcialmente en los resultados de los trabajos que abordan la planificación de la fecundidad, que cuentan con una amplia tradición en este sentido. 
Todas estas razones apoyan la necesidad de entender el tiempo como algo fluido (véase en Saldaña 2003) y multidimensional (Caïs et al. 2014). Más precisamente, Saldaña (2003: 5) a través de varios ejemplos explica que el tiempo no es solamente un constructo físico, sino también cultural, que puede ser interpretado individualmente y subjetivamente, ya que la gente vive el tiempo de acontecimientos y de fases en su flujo. Parecidamente, Caïs et al. (2014) discuten múltiples maneras de cómo entender el tiempo y añaden que el tiempo puede ser abarcado también como un recurso o como una barrera que se debe gestionar en una multitud de contextos. Los autores finalmente explican que "Entender cómo la gente evoluciona a lo largo del tiempo, cómo utiliza el tiempo, y cuáles son sus estrategias para dar sentido al pasado y así ser capaces de definir el presente y programar el futuro, requiere una comprensión profunda de las circunstancias individuales de la vida diaria de las personas." (Caïs et al. 2014: 16) Entonces, para entender e identificar los mecanismos causales que conectan la fase pre-decisoria (intencional) con la acción de retornar / asentarse / re-emigrar o circular entre ambos países es imprescindible que los datos tengan un carácter temporal. Según Thomson (2007) este tipo de datos donde la especificidad, los acontecimientos concretos, las yuxtaposiciones y las coincidencias, se encuentran en el corazón de la historia analítica ayudan a superar una simple descripción cronológica de acontecimientos y de cambios, permitiendo una exploración más dinámica del cómo el individuo está involucrado en la creación de ese cambio, en un contexto temporal y social específico.

Son los estudios longitudinales cualitativos que recopilan los datos de forma prospectiva mediante los cuales se pueden explorar los procesos causales de la temporalidad de los sucesos (Holland 2007), que parecen ser predilectos para abordar todo el proceso intencional y decisorio como un continuo y ayudar comprender mejor las dinámicas del fenómeno. Según Farral (2006), por un lado, la investigación longitudinal cualitativa destaca sobre la investigación longitudinal cuantitativa en el hecho de que permite explorar contextos, mecanismos y resultados a nivel individual. Por otro lado, tiene ventaja sobre la investigación cualitativa transversal, ya que permite captar y comprender mejor los cambios en la vida de los actores que pueden alterar sus experiencias, y así visibilizar mejor los impactos de unos eventos en diferentes puntos del tiempo. Sin embargo, como constata Winiarska (2017) hasta la fecha la perspectiva longitudinal cualitativa, aunque potencialmente adecuada, no ha recibido mucha atención en el campo de la migración intencional.

Así, el presente artículo contribuye a rellenar esta laguna a través de una reflexión sobre estudio de caso de los ucranianos en la Comunidad de Madrid, cuyo objetivo ha sido entender cómo se construyen, modifican y mantienen las intenciones/decisiones migratorias a lo largo del tiempo en un contexto de incertidumbre. El caso ucraniano en España es particularmente interesante, dada la cambiante y compleja situación que resulta de la conjunción entre la crisis económica en el país de destino (España) y el conflicto económico, social y armado en el país de origen (Ucrania). En este estudio el proyecto migratorio ha sido abordado como un proceso dinámico que está en continua interrelación 
con el entorno relacional de los individuos y con el contexto macroestructural. Es decir, se han reconstruido tres ejes temporales donde: Eje 1) representa la evolución del proyecto migratorio y de las motivaciones migratorias insertadas en las trayectorias vitales; Eje 2) representa el tiempo contextual / histórico que tiene en cuenta la evolución de contextos sociopolíticos en el país de destino y en el país de origen y que se traduce en diferentes niveles de inseguridad; Eje 3) representa la evolución del entorno relacional que está evaluado mediante un análisis longitudinal de las reconfiguraciones espaciales y temporales de las redes personales de los entrevistados. Cabe mencionar que el papel de las redes sociales en el proyecto migratorio ha atraído un interés creciente en las últimas dos décadas, particularmente en lo que respecta a la fase inicial de la emigración desde el país de origen (p. ej., Portes y Sensenbrenner 1993). Sin embargo, la literatura, que presta especial atención a las redes sociales y sus impactos en el proceso intencional / decisorio de asentarse o partir para aquellos que ya se encuentran en el país de destino, es limitada (p. ej., Stamm 2006). Curiosamente, la mayoría de estos estudios utilizan la teoría de las redes de migración con el concepto de capital social como herramienta analítica. Entre ellos, por ejemplo, Boyd (1989) y Fawcett (1989) han señalado que las redes sociales son un determinante importante de los planes de migración; o Gurak y Caces (1992) han destacado la importancia de los débiles vínculos de los migrantes en este proceso. Sin embargo, pocos autores abordan este fenómeno desde la perspectiva del análisis de las redes sociales que permite una recogida de datos relacionales sistemática y un análisis exhaustivo de impacto de relaciones en el proceso de las intenciones residenciales. Además, pocos estudios plantean la importancia de estudiar el entorno relacional como un resultado, más que como un determinante (véase Tsuda 1999), o destacan la reciprocidad entre la red social y la migración (véase Brown 2002). Entonces, entre los propósitos de este estudio ha sido llegar a confrontar los tres ejes temporales mencionados, y así intentar descifrar procesos causales entre ellos.

Los datos del estudio (a partir de aquí, "UCRAM 2009-2016"2) fueron recogidos en distintas oleadas entre los años 2009 y 2016. La primera oleada se realizó en el año 2009 en la Comunidad de Madrid. En los años 2012 y 2015 se hizo el seguimiento de intenciones / decisiones / acciones migratorias en y desde la Comunidad de Madrid. Es decir, que con aquellos entrevistados que se habían mudado a otra comunidad en España o retornaron a Ucrania realizamos las entrevistas exclusivamente por teléfono y/o Skype ${ }^{3}$. En el año 2016, con una

${ }^{2}$ El estudio UCRAM 2009-2016 fue financiado en gran parte por el Consejo Superior de Investigaciones Científicas - CSIC (beca predoctoral I3P) y apoyado por los siguientes proyectos y grupos: CSO2011-29970, CSO200806130/SOCI, GEPS S2007/HUM-0517 y Unidad Asociada Estudios Demográficos y Sociales. Su ampliación postdoctoral fue apoyada por "Excellence Laboratory" "Structuring of Social Worlds" (SMS, ANR-11-LABX-0066) como parte de un programa del Estado francés (ANR-11-IDEX-0002-02).

${ }^{3}$ El estudio UCRAM 2009-2016 contiene una muestra aparte de migrantes retornados que fueron entrevistados en el año 2010 en Ucrania en distintas regiones. Sin embargo, estos datos no están discutidos ni analizados en el presente artículo, ya que no forman parte del seguimiento 
submuestra, se hizo un seguimiento longitudinal de redes personales junto a las intenciones / decisiones migratorias. En los distintos lugares en la Comunidad de Madrid realizamos, primero un muestreo aleatorio (intentando aproximarse a la composición real de la población ucraniana) y posteriormente implementamos la técnica de bola de nieve para la búsqueda de los perfiles específicos identificados durante el desarrollo del trabajo de campo. Es decir, se ha intentado seguir, por un lado, el criterio estadístico, y por el otro, el criterio analítico. La muestra principal (del año 2009) contiene 26 hombres y 27 mujeres, de edades comprendidas entre los 20 y los 65 años (con una media de 35 años), con tiempo de residencia en España de entre 1 y 11 años (con una media de 6 años). 27 $(51 \%)$ de los casos llegaron a España teniendo el nivel de estudios universitario, 32 sujetos $(60 \%)$ estaban empleados cuando se realizó la entrevista y 40 (75\%) se encontraban en la situación legal en España. A la mitad de ellos se les puede considerar como primo-migrantes (dentro de la cadena migratoria familiar). En cuanto a lugar de residencia 47\% residían en Madrid capital, 22\% en los municipios con alta concentración de ucranianos y $32 \%$ en los municipios con media y baja concentración de ucranianos.

El propósito del presente artículo es evaluar la capacidad del enfoque longitudinal cualitativo para comprender el proceso del proyecto migratorio y para discernir la relación causal entre él, los factores macroestructurales y el entorno relacional de los entrevistados. Se trata de presentar no solamente las aportaciones, sino también las dificultades y los retos conceptuales, metodológicos y analíticos de este enfoque para el estudio del proceso intencional / decisorio en migraciones internacionales ${ }^{4}$.

Entonces, a continuación presentaremos el diseño y conceptualización del estudio, discutiendo los retos con los que nos hemos encontrados. Después, expondremos los elementos claves y los desafíos que el investigador debe tener en cuenta para la ejecución del trabajo de campo. Finalmente, presentaremos las opciones y contribuciones analíticas, discutiendo algunos de los resultados de la investigación.

\section{DISEÑO Y CONCEPTUALIZACIÓN DEL ESTUDIO}

\subsection{Operacionalización del proyecto migratorio y el arte de preguntar}

Los trabajos sobre intenciones y decisiones migratorias evidencian la existencia de distintos usos y definiciones de la intención y una confusión sobre lo

\footnotetext{
longitudinal.

${ }^{4}$ En realidad, UCRAM 2009-2016 es un estudio longitudinal basado en un "diseño de método mixto" donde se han utilizado tanto los métodos cualitativos como cuantitativos para la recogida y análisis de los datos. Aunque en este artículo nos centramos en la parte cualitativa del estudio, consideramos importante hacer referencia también a la parte cuantitativa para ofrecer una mejor comprensión del estudio y las aportaciones de la perspectiva cualitativa longitudinal para el estudio del fenómeno.
} 
que engloba la fase intencional y la fase de toma de decisiones, ya que existe poca claridad y coherencia conceptual sobre términos como planes, deseos, expectativas e intenciones (McHuhg 1984, Sell y De Jong 1983). Como Kodzi et al. (2010) advierten, el grado de compromiso del individuo hacia el comportamiento no es igual para todos los conceptos mencionados. Por ejemplo, según Van Dalen et al. (2005) la voluntad (el deseo) de migrar no está asociada a una planificación o a acciones orientadas a tal fin como en el caso de la intención. Por lo tanto, antes de diseñar los instrumentos de recogida de datos, una reflexión y diferenciación entre los conceptos mencionados es clave puesto que la formulación de la(s) pregunta(s) y el nivel de comprensión del entrevistado determinan la(s) respuesta(s), lo cual luego influye en los resultados. También, hay que pensar en que las respuestas pueden variar en función del tipo de pregunta, de si y cómo se detalla el lugar del cambio de residencia intencionado; y de su temporalidad, es decir, de si y cómo se determinan los horizontes temporales.

Con respecto a la acción de retorno, ésta es algo más clara conceptualmente, ya que contempla aspectos mejor observables y basados en los hechos. El retorno efectivo (sea definitivo o no) puede ser entendido de forma más estricta y acotada como un mero traslado residencial desde el país de destino al país de origen, o en sentido más amplio, entendido desde la toma de decisión, tras la preparación del retorno hasta el retorno mismo. Sin embargo, ni siquiera el concepto de acción de retorno está totalmente libre de ambigüedades. En la literatura podemos encontrar distintas definiciones y tipos de retorno (véase p. ej. en Cerase 1974, Durand 2004), y según qué lentes teóricos se utilicen el retorno puede ser tratado o bien como una parte o bien como el punto final del proyecto migratorio. Si ya en la segunda mitad del siglo XX no siempre fue posible distinguir entre aquellos que retornaron permanentemente y aquellos que realizaban visitas al país de origen (véase Gmelsch 1980), hoy en día, donde la migración circular o la re-emigración es cada vez más frecuente, esta distinción se hace aún más difícil. Una de las soluciones a este problema es introducir en la definición del retornado el elemento que refleje la duración intencional del retorno, en vez de contar con retornos efectivos (Hosnedlova 2014). Luego, también es importante que el investigador indague sobre ciertos aspectos (el intento de retornar y las estrategias de retorno) que implican acción durante el proceso migratorio, pero que se encuentran en la frontera entre la fase intencional y la fase de la acción.

Asimismo, es necesario recalcar que la intencionalidad / el proceso decisorio, siendo parte del proyecto migratorio, engloba también la opción del asentamiento, de re-emigración a un tercer país y la indecisión. Por lo que también se puede dar la acción de asentamiento o acción de re-emigración. Si bien, la acción de retorno o de re-emigración son unos hechos más fácilmente observables, la determinación del asentamiento definitivo no lo es tanto, ya que se trata de un proceso donde es difícil marcar la frontera entre el alcance de las estrategias e intentos del asentamiento definitivo y la noción de estar asentado definitivamente. Aunque con una batería de preguntas cerradas bien diseñadas se pueda llegar a captar las futuras intenciones / decisiones residenciales, es la aproximación 
cualitativa la que nos ayudará a comprender este fenómeno como un proceso dinámico, desvelando los matices que subyacen en las respuestas.

Ahora bien, para que la información conseguida en distintas oleadas sea comparable, incluso en un estudio longitudinal cualitativo la manera de indagar sobre los aspectos claves debería ser la misma. Es decir, hay que repetir la misma manera de preguntar sobre las intenciones y decisiones migratorias en todas las oleadas. Aunque en las oleadas siguientes se puedan añadir otras preguntas para poder profundizar sobre las causas / motivaciones del cambio en el proyecto migratorio y abordar aspectos que han surgido de manera inesperada. De hecho, una de las habilidades clave de la aproximación cualitativa en un estudio longitudinal es una cierta flexibilidad y capacidad de respuesta a los datos y los análisis emergentes (Calman et al. 2013) lo cual permite delimitar y centrarse en los procesos significativos en el proyecto migratorio del individuo. Sin embargo, no hay que olvidar que es importante el momento en el que se realicen preguntas sobre las intenciones y decisiones, ya que se trata de preguntas que son hipotéticas y sus respuestas se basan en las percepciones y evaluaciones de las experiencias, los actos pasados y la situación presente del entrevistado. Si se le pregunta al entrevistado sobre cómo ve su futuro después de una narración con un contenido negativo, puede que las respuestas estén influenciadas por la mala experiencia anteriormente narrada. Asimismo, podría influir en el ánimo del entrevistado el día y el momento de la entrevista. Por las razones expuestas y para no perder riqueza informativa, en la primera oleada, se siguió con la estrategia de obtener una buena narrativa sobre la formación de las intenciones y el proceso decisorio, utilizando varias preguntas (7) sobre el futuro plan migratorio a cada entrevistado, y dialogando sobre ellas para indagar sobre el grado de compromiso. Esta estrategia resultó muy fructífera no sólo para conseguir las respuestas requeridas y observar cómo cambia la respuesta con cada pregunta, pero también para detectar las posibles causas que pueden influir en el proceso decisorio. Por otro lado, en una estrategia para comprobar la validez interna de los datos (Siedman 1998), se incluyó una batería de tres preguntas cerradas en el cuestionario que se aplicó al final de la primera fase de entrevista. La pregunta sobre las intenciones se delimitó temporalmente, interrogando sobre las intenciones residenciales en tres horizontes temporales (a corto, a medio y a largo plazo): ¿Qué intenciones tiene usted para el próximo año? / los próximos 5 años? / los próximos 15 años? El entrevisto tuvo que elegir una de cuatro posibles respuestas: 1.) Retornar a Ucrania; 2.) Permanecer en España; 3.) No tener intenciones claras; o 4.) Reemigrar a otro país. Además, esta misma batería de preguntas se repitió durante el segundo encuentro (unos pocos días después) con el entrevistado para ver la consistencia de la respuesta, y así descartar el sesgo que pudiera ocasionar la influencia del ánimo del entrevistado en el momento de los encuentros.

Varios estudios subrayan que en un seguimiento longitudinal cualitativo la riqueza de información recogida puede ser hasta abrumadora, lo cual obstaculiza un análisis en profundidad de todas las entrevistas (Calman et al. 2013; Wray et al. 2007). Por esta razón, algunos investigadores recomiendan realizar un análisis preliminar y resumen de cada entrevista antes de empezar la siguiente 
oleada para poder empezar la entrevista de seguimiento con una recapitulación de la última narración y hacerle al entrevistador reflexionar sobre ella (Calman et al. 2013). Esta estrategia, durante las entrevistas de seguimiento, nos ayudó a centrarnos directamente en los aspectos vinculados al proyecto migratorio que nos interesaban analizar. A continuación, para que los datos fueran comparables entre todas las oleadas, se repitió la misma batería de preguntas cerradas sobre las intenciones, utilizada en la primera oleada, a todos los individuos localizados. También se repitieron las mismas preguntas en cuanto a las estrategias llevadas a cabo en relación con su proyecto migratorio. A aquellos que habían retornado y/o re-emigrado se les preguntó, además, sobre el período y los motivos del cambio de país de residencia y sobre las acciones y estrategias llevadas a cabo para poder realizar la movilidad. Aquí es necesario puntualizar que, en nuestro trabajo, para poder distinguir entre las visitas y el retorno o la re-emigración, una persona retornada (o re-emigrada) ha sido aquella que había permanecido al menos un año en España y retornó (re-emigró) a su país (a otro país) con la intención de permanecer allí al menos un año. También contábamos con la posibilidad de una nueva re-emigración a España.

\subsection{La importancia de bien establecer los períodos de observación}

Toda investigación longitudinal necesita de una reflexión sobre cómo espaciar las oleadas de observación, lo cual depende de los objetivos de investigación, la población, el proceso del fenómeno estudiado, y las posibilidades de investigación. A pesar de que no existan directrices sobre la duración del estudio longitudinal, sobre el número ni espaciamiento de las oleadas, según nuestra experiencia es aconsejable que los intervalos entre cada oleada no sean ni demasiado cortos ni demasiado largos, y estén igualmente espaciados. Por una parte, hay que dejar tiempo suficiente para poder captar y evaluar la evolución del proyecto migratorio, lo cual depende de la formulación de la pregunta sobre las intenciones y el proceso decisorio durante el trabajo de campo. Además, si se pretende captar la influencia de los factores macro-estructurales y contextuales, los momentos de recogida de datos deberían elegirse en función de la situación y/o los cambios contextuales que no siempre son predecibles y esperados. Luego, hay que tener en cuenta que se trata de una población móvil que además suele encontrarse en situación de precariedad e instabilidad, lo cual implica necesariamente que dejar períodos demasiado largos entre cada oleada aumentaría la probabilidad de la disminución de la muestra.

Como ya hemos mencionado en la introducción, en nuestro caso la recogida de los datos se llevó a cabo en tres oleadas principales (en 2009, 2012 y 2015), y una oleada complementaria (2016) donde nos centramos sólo en una sub-muestra de casos. Cada período de observación representa un contexto macroestructural distinto: 1.) En el año 2009, en España se empiezan a notar los efectos de la crisis económica; 2.) Entre los años 2009 y 2012, la crisis económica en España empeora y alcanza sus peores niveles, y en Ucrania en el año 2010 el candidato 
pro ruso Viktor Yanukovych gana las elecciones presidenciales, lo cual agrava el clima social, económico y político; 3.) Entre los años 2012 y 2015, la crisis económica en España sigue siendo presente, y además se añade el contexto del conflicto armado en Donbass, que se desencadenó tras las protestas pro-europeas en 2013 y 2014 y la crisis de Crimea; 4.) Entre el año 2015 y 2016, la situación en Ucrania cambia ligeramente, gracias a que las oleadas de conscripciones que aterrorizaban a muchos ucranianos (residentes tanto en Ucrania como en el extranjero) han cesado tras la creación de un ejército profesional. Así, los cuatro períodos de observación representan distintos tipos y niveles de inseguridad en los lugares de destino y de origen para el migrante ucraniano.

Entonces, la temporalidad de intervalos de recogida de datos y la duración del estudio es un resultado de la búsqueda de un equilibrio entre todos los aspectos mencionados.

\section{EJECUCIÓN DEL TRABAJO DE CAMPO LONGITUDINAL}

\subsection{Herramientas utilizadas para el seguimiento longitudinal}

El trabajo de campo principal se realizó en el año 2009 en la Comunidad de Madrid que tuvo dos fases. Durante la primera fase se realizó una entrevista semi-estructurada basada en un guión esquemático que contenía tres grandes bloques: 1.) Trayectoria migratoria; 2.) País y cultura de origen, país de acogida, sentido de pertenencia, futuro, retorno; 3.) Familia y amistades. El objetivo era conseguir información sobre la trayectoria migratoria del entrevistado, sobre sus intenciones, motivaciones, decisiones, estrategias y actitudes. Después de cada entrevista se aplicó un cuestionario corto con el fin de unificar la información socio-demográfica básica y las intenciones residenciales de todos los entrevistados. La segunda fase tuvo la forma de entrevista-encuesta y su objetivo fue construir la red personal de cada entrevistado. La red se construyó con la ayuda del software EgoNet ${ }^{5}$ que, entre otras virtudes, permitió visualizar las redes en el acto. Esto dio la oportunidad de poder reflexionar con cada entrevistado sobre su propia red, e indagar más sobre su entorno relacional a nivel cualitativo. Para construir la red personal se utilizó un generador de nombres múltiple que consistió en tres preguntas que abarcaron tanto a la familia, como a los amigos y otros contactos relevantes. El número de los contactos a recolectar fue fijo, establecido a 30, que es el mínimo requerido para que las características estructurales de las redes sean comparables entre sí y en el tiempo (véase McCarty y Killworth 2007). A nivel de relaciones, se tuvieron en cuenta los vínculos fuertes, débiles y negativos (un tipo de relaciones normalmente ignorado, a pesar de que algunos estudios demuestran su importancia (p. ej. Hoang 2011)).

${ }^{5}$ McCarty, C., 2003. EgoNet. Personal Network Software. Disponible en: http://sourceforge. net/projects/egonet/ Versión 2009-05-30 
En 2012 y 2015, tres y seis años después de la realización de la primera oleada de entrevistas se intentó establecer contacto con todos los entrevistados para averiguar si han permanecido en España o no. Con ello, se pretendió evaluar la repercusión de la intención sobre la acción. Como ya hemos explicado, durante estas entrevistas de seguimiento se les interrogó de nuevo sobre las intenciones en tres horizontes temporales (en 1, 5, y 15 años), con el fin de indagar sobre la estabilidad de las intenciones en el tiempo. Además, a aquellos ucranianos que habían permanecido en España se les hicieron varias preguntas relacionadas con los motivos para o causas de (no) retornar / asentarse permanentemente / re-emigrar y sobre sus futuras expectativas. A aquellos que cambiaron de intenciones con respecto a la oleada anterior, se les preguntó por los motivos del cambio. A aquellos que retornaron / re-emigraron se les preguntó sobre la fecha de retorno / re-emigración, sobre las circunstancias y motivos de retorno. A parte, con todos los entrevistados se ha discutido sobre su evolución de su situación laboral, sobre su entorno relacional en general, y sobre las condiciones de sus familias en particular.

En el año 2016, se entrevistó una submuestra de individuos para el seguimiento longitudinal de redes personales. El objetivo primario de esta oleada fue mapear la evolución de la composición y de la estructura de las redes personales de los individuos después de 7 años, que engloban distintos niveles de inseguridad contextual para, luego, profundizar sobre la interrelación entre el proyecto migratorio y el entorno relacional ya demostrada a partir de la primera oleada. Entonces, a los entrevistados se les aplicó el mismo cuestionario de redes personales que en la primera oleada. No obstante, se añadieron algunas preguntas para conocer las causas de la disolución y/o de reemplazamiento de los vínculos sociales. Luego, se realizaron entrevistas en profundidad para comprender las dinámicas de la evolución de redes personales, de los proyectos migratorios y la relación entre ellos.

Por lo tanto, esta última oleada permitió poner en relación los acontecimientos de los tres ejes temporales observados, pretendiendo así arrojar luz sobre el proceso causal entre las intenciones/decisiones, el entorno relacional y los cambios macroestructurales.

\subsection{Construcción de la muestra longitudinal y la problemática de seguimiento de los entrevistados}

En general, en los estudios cualitativos se les otorga menos espacio a la problemática del muestreo que en los estudios cuantitativos. Empero, una reflexión profunda sobre lo que debe ser la muestra y sus fines en un estudio cualitativo es también requerida, y eso aún más, cuando se trata de un estudio longitudinal donde la reducción de la muestra en el tiempo es casi inevitable. Es más, en relación con el fenómeno abordado en el presente artículo hay que tener presente que los migrantes son especialmente propensos al sesgo de auto-selección que viene determinado por la situación específica social, política y económica 
en la cual el migrante se encuentra (Winiarska, 2017). Por otro lado, se puede esperar que la probabilidad de pérdida de cierto perfil de entrevistado a lo largo de estudio será mayor que del otro (por ejemplo, encontrar y entrevistar a los retornados puede ser más problemático que entrevistar a los inmigrantes que no cambiaron de lugar de residencia). Por estas razones, en un estudio longitudinal con una aproximación cualitativa siempre es importante diseñar bien el método de muestreo de la primera oleada porque ésta va a influenciar las siguientes. Es decir, es requerido decidir el grado de homogeneidad de perfiles, el tamaño de la muestra, la estrategia del muestreo y las fuentes de reclutamiento, estando todos estos aspectos acordes a los objetivos del estudio.

En nuestro caso, cuando empezamos a diseñar la investigación no habíamos encontrado estudios que abordasen el proyecto migratorio como un proceso desde una perspectiva holística teniendo en cuenta la interacción de tres niveles contextuales: micro (individual) - meso (relacional) - macro (macroestructural). Tampoco, existían muchos estudios sobre la población ucraniana en España que nos pudieran aportar pistas sobre el fenómeno de toma de decisiones residenciales para la construcción de la muestra. Entonces, con el fin de conocer las características del universo de estudio, a la hora de construir la muestra, seguimos, por un lado, el criterio estadístico - intentando aproximarse a la composición real de la población ucraniana en la Comunidad de Madrid en cuanto a los indicadores básicos (índice de masculinidad, composición según la edad, distribución territorial - distinguiendo los municipios con alta, media y baja concentración de ucranianos; tasa de irregularidad) extraídos del Padrón de Habitantes y otras estadísticas oficiales. Por otro lado, se empleó la estrategia de selección orientada hacia la información (es decir, el criterio analítico), pero siempre procurando cubrir los cuatro indicadores de composición de la muestra indicados anteriormente. El interés fue encontrar casos con máxima variación en cuanto al tiempo de residencia y situación familiar. Con ello, se trató de obtener información sobre la significación de varias circunstancias en la evolución y consecuencias del proyecto migratorio pasado, presente y futuro. Además, se buscaron los casos extremos con la esperanza de que pudiesen ayudar a aclarar la lógica de ciertos sucesos. Con respecto a la búsqueda de los entrevistados, se identificaron diversas fuentes para asegurar una gran heterogeneidad y reducir el riesgo de superposición de las redes personales. En los distintos lugares (fuentes) realizamos, primero un muestreo aleatorio y posteriormente implementamos la técnica de bola de nieve para la búsqueda de los perfiles específicos identificados durante el desarrollo del trabajo de campo. Establecimos el mínimo de los individuos a entrevistar en 50, pensando tanto en la posibilidad de realizar los test estadísticos como en la saturación teórica y el poder tratar la cantidad de información cualitativa recogida. Durante las siguientes oleadas (en 2012 y 2015) intentamos localizar y entrevistar el máximo posible de individuos entrevistados en al año 2009.

La tabla 1 sintetiza las características básicas de la muestra y su evolución en el tiempo. En la oleada inicial contamos con 53 entrevistados. Tres años después conseguimos entrevistar a 43 de 51 localizados, y 6 años después del comienzo 
del estudio 31 entrevistados (de 39 localizados) aceptaron a participar en la $3^{\text {era }}$ oleada.

Tabla 1. Muestra y su evolución según las tres oleadas principales

\begin{tabular}{|c|c|c|c|c|c|c|}
\hline $\begin{array}{l}\text { Año de } \\
\text { la olea- } \\
\text { da }\end{array}$ & $\begin{array}{l}\text { Entrevistados } \\
(\mathrm{N})\end{array}$ & Sexo & $\begin{array}{l}\text { Edad } \\
\text { min- } \\
\text { max } \\
\text { (media) }\end{array}$ & $\begin{array}{l}\text { Años de } \\
\text { residen- } \\
\text { cia en } \\
\text { España } \\
\text { min- } \\
\text { max } \\
\text { (media) }\end{array}$ & $\begin{array}{l}\text { Con permi- } \\
\text { so de resi- } \\
\text { dencia y/o } \\
\text { trabajo (N/ } \\
\text { de total) }\end{array}$ & $\begin{array}{l}\text { Familia } \\
\text { uni-local } \\
\text { / Familia } \\
\text { multi-lo- } \\
\text { cal / Sin } \\
\text { familia } \\
\text { inmediata }\end{array}$ \\
\hline 2009 & 53 & $\begin{array}{l}26 \mathrm{~m} \\
/ 27 \mathrm{f}\end{array}$ & $\begin{array}{l}20-65 \\
(35)\end{array}$ & $1-11(6)$ & $40 / 53$ & $28 / 12 / 13$ \\
\hline 2012 & $\begin{array}{l}43 \\
\text { (de } 51 \text { localiza- } \\
\text { dos) }\end{array}$ & $\begin{array}{l}18 \mathrm{~m} \\
/ 25 \mathrm{f}\end{array}$ & $\begin{array}{l}23-68 \\
(39)\end{array}$ & 4-14 (9) & $40 / 43$ & $29 / 7 / 7$ \\
\hline 2015 & $\begin{array}{l}31 \\
\text { (de } 39 \text { localiza- } \\
\text { dos) }\end{array}$ & $\begin{array}{l}12 \mathrm{~m} \\
/ 19 \mathrm{f}\end{array}$ & $\begin{array}{l}26-68 \\
(42)\end{array}$ & $\begin{array}{l}7,5-16 \\
(12,5)\end{array}$ & $30 / 31$ & $24 / 4 / 3$ \\
\hline
\end{tabular}

La evolución de nuestra muestra se aproxima a algunas de las pautas de evolución de la población ucraniana en la Comunidad de Madrid (por ejemplo, el índice de masculinidad y la tasa de irregularidad disminuyen, el tiempo de residencia aumenta). No obstante, hay otras características de la muestra que se deben al fenómeno de autoselección y que hubo que tener en cuenta durante los análisis. Por ejemplo, en la muestra se han mantenido más aquellos casos con mayor nivel educativo (mientras que la primera oleada contiene $51 \%$ de individuos con estudios universitarios, en la tercera oleada esta proporción aumenta a $65 \%)$.

En cuanto a la cuarta oleada, decidimos seleccionar una sub-muestra en función de los perfiles analíticos y las disponibilidades para esta última oleada. Durante las entrevistas de la tercera oleada sondeamos la disposición y la disponibilidad de los entrevistados para participar en la cuarta oleada, explicando los objetivos y la duración de la entrevista (alrededor de 2h). De los 31 entrevistados 10 rechazaron por cuestiones de tiempo y/o por desinterés en discutir sobre su entorno relacional. Previendo que la disponibilidad de los que habían aceptado podía aún cambiar para el año siguiente, decidimos preseleccionar unos perfiles (descritos en la página siguiente) que tenían que estar incluidos en la submuestra de esta última oleada. Para ello, realizamos primero un análisis exploratorio de la evolución de las intenciones y acciones residenciales entre los años 20092012-2015 y aprendimos que: 1.) Los ucranianos a menudo redefinen sus planes residenciales, incluso si se trata migrantes de larga residencia (véase la figura 1); 
2.) Fue posible distinguir grupos con distintos itinerarios intencionales; 3.) Había que recentrar una parte de los análisis, prestando menos atención al retorno e intentando explicar la formación de intenciones/decisiones de asentarse a largo plazo / definitivamente en al país de destino. Si bien en un estudio cualitativo transversal la heterogeneidad de la muestra es una característica de gran interés, en un estudio cualitativo longitudinal es sobre todo la trayectoria y el cambio en los eventos lo que gana importancia (Calman et al. 2013). Es decir, es aconsejable buscar una representatividad de sujetos y la posibilidad de comparar entre sí a partir de una muestra orientada a la "profundización en el contraste"6 (Pirès 1997).

\section{Figura 1. Secuencias de las intenciones expresadas a largo plazo por cada entrevistado}

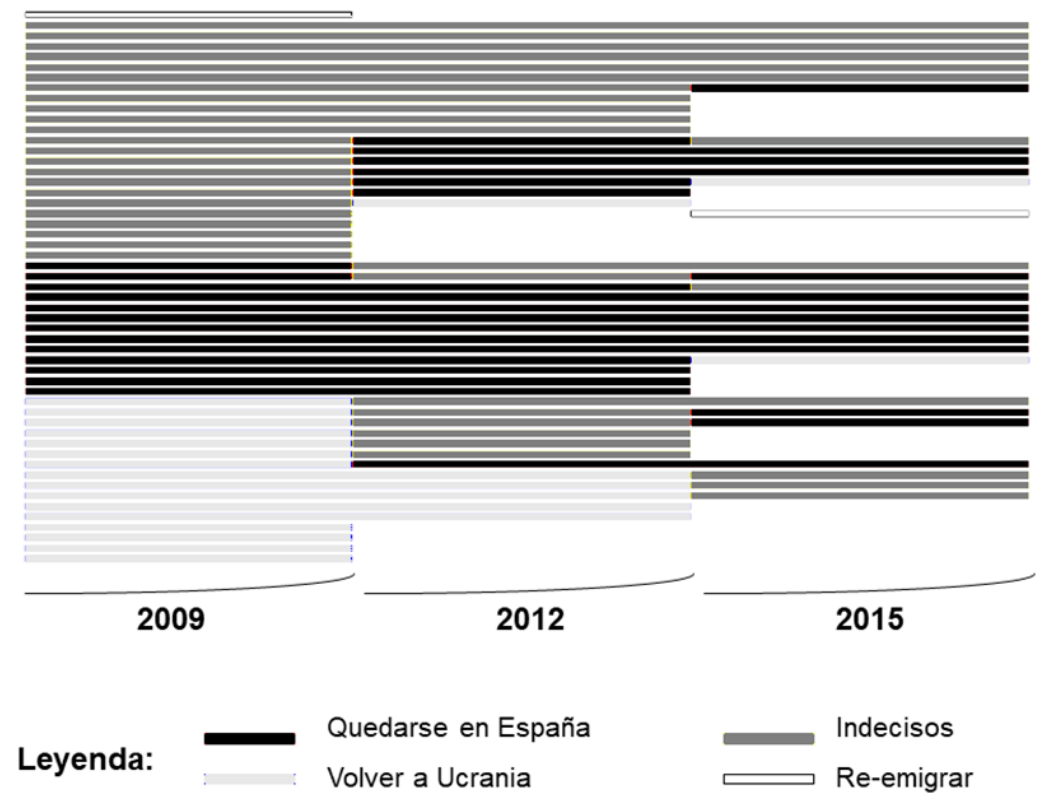

Entonces, teniendo en cuenta las tres oleadas identificamos cuatro perfiles de itinerarios intencionales ("permanente confirmado", "recién permanente", "indeciso", "cambiante" véase la tabla 2). Por lo que el objetivo de la sub-muestra de la última oleada fue tener los 4 perfiles representados, y además, incluir algunos casos atípicos en cuanto a la evolución del proyecto migratorio y/o situación

${ }^{6}$ Traducción propia del francés (l'échantillon par « contraste-approfondissement »). 
familiar. De este modo, primero nos centramos en entrevistar 8 personas cumpliendo con estas características (al menos 2 entrevistados en cada perfil, siendo un caso típico y otro atípico) y fuimos completando la muestra hasta agotar la disponibilidad de los otros posibles participantes. Así, obtuvimos una submuestra de 13 entrevistados.

Tabla 2. Proceso de selección de casos para la $4^{\text {a }}$ oleada y su composición

\begin{tabular}{|c|c|c|c|c|c|c|c|}
\hline & \multicolumn{3}{|c|}{ Intenciones constantes } & \multicolumn{3}{|c|}{ Intenciones modificadas } & \multirow[b]{2}{*}{$\begin{array}{l}\text { Total } \\
(\mathrm{N})\end{array}$} \\
\hline & $\begin{array}{l}\text { Permanecer } \\
\text { en España }\end{array}$ & $\begin{array}{l}\text { Retornar a } \\
\text { Ucrania }\end{array}$ & Indecisión & \begin{tabular}{|c|}
$\begin{array}{c}\text { Modificación } \\
\text { entre } 2009 \text { y } \\
2012 \\
\end{array}$ \\
\end{tabular} & \begin{tabular}{|c|}
$\begin{array}{c}\text { Modificación } \\
\text { entre } 2012 \text { y } \\
2015\end{array}$ \\
\end{tabular} & \begin{tabular}{|c|} 
Modificación \\
en 2012 y \\
2015 \\
\end{tabular} & \\
\hline $\begin{array}{l}1-2 \\
\text { oleadas }(\mathrm{N})\end{array}$ & 11 & 5 & 11 & 16 & & & 43 \\
\hline $\begin{array}{l}1-2-3 \\
\text { oleadas }(\mathrm{N})\end{array}$ & 7 & & 6 & 6 & 7 & 5 & 31 \\
\hline $\begin{array}{l}\text { Itinerario } \\
\text { intencional } \\
(\mathrm{N}) \\
\end{array}$ & $\begin{array}{c}\text { Permanente } \\
\text { confirmado } \\
(7) \\
\end{array}$ & & Indeciso (6) & \multicolumn{3}{|c|}{$\begin{array}{l}\text { Recién permanente ( } 8) \\
\text { Cambiante (10) }\end{array}$} & \\
\hline $\begin{array}{l}4^{\mathrm{a}} \text { oleada } \\
(\mathrm{N})\end{array}$ & $\begin{array}{l}\text { Permanente } \\
\text { confirmado } \\
\text { (5) }\end{array}$ & & Indeciso (2) & \multicolumn{3}{|c|}{$\begin{array}{l}\text { Recién permanente (4) } \\
\text { Cambiante (2) }\end{array}$} & 13 \\
\hline
\end{tabular}

El seguimiento de los entrevistados representa un verdadero desafío en los estudios longitudinales. Varios autores aconsejan diversas estrategias para evitar un excesivo "adelgazamiento" de la muestra en el tiempo. Entre los más importantes es mantener: una hoja de contacto de los entrevistados actualizada; el contacto activo con el entrevistado; y la confianza en el estudio, indicando cuál es su contexto institucional y financiación, explicando la importancia y insustituibilidad de la participación de cada entrevistado (véase p. ej. en Farral et al. 2016). No obstante, ningún autor especifica cuál es el tiempo adecuado para volver a ponerse en contacto con el entrevistado. Nosotros, para evitar ser demasiado intrusivos decidimos re-contactar a todos los entrevistados a principio de cada oleada para informar sobre la continuación del estudio y acordar una entrevista. Esto funcionó bien con los entrevistados que conseguimos localizar "a la primera", y la reacción en la mayoría de los casos fue positiva. Sin embargo, debemos constatar que un período de tres años es quizás demasiado largo tratándose de una población in/migrante, ya que una parte importante de los entrevistados cambió de número de teléfono, de residencia (bien a nivel nacional o bien a nivel internacional), o su situación personal (p. ej. se divorció). Para localizar a estos entrevistados nos apoyamos en los informantes claves y medios 
sociales de comunicación (Facebook, LinkedIn, etc.). Nuestra experiencia es que cuanto más diversa información de contacto se tiene sobre cada entrevistado (teléfono, email, cuenta skype, nombre completo), mejor. La mayoría de las entrevistas de seguimiento se hicieron por teléfono, excepto para aquellos que habían retornado. Para estos se buscaron otras vías para conocer los motivos y fecha de retorno. Si bien, una parte pudiera ser contactada directamente a través de - email o Skype, una parte de la información sobre los retornados se obtuvo a través de contactos indirectos (a través de informantes clave y a través de otros inmigrantes ucranianos aún residentes en España y que eran conocidos de estos retornados). Por el contrario, las entrevistas de la cuarta oleada, siendo más extensas, se realizaron en persona.

\section{APORTES Y RETOS ANALÍTICOS PARA EL ESTUDIO DEL FENÓMENO INTENCIONAL / DECISORIO MIGRATORIO}

El proceso analítico en un estudio cualitativo longitudinal, que implica trabajar al mismo tiempo con datos de escala pequeña (en términos de número de casos) y escala grande (en términos de la intensidad de los datos acumulados para cada individuo), requiere un alto grado de reflexividad, cuidadosa interpretación y fundamentación empírica en un campo teórico más amplio (Yates 2003). Los datos son especialmente exigentes en cuanto a las tareas de organización y codificación, las cuales en sí posibilitan los primeros análisis exploratorios. Durante el proceso que va desde el almacenamiento y categorización de los datos hasta su análisis, el investigador tiene que ser creativo y flexible, pero al mismo tiempo riguroso, perseverante y siempre sincero con los datos recogidos. Dada la naturaleza de nuestros datos (cuali-cuantitativos) elegimos almacenar y analizarlos con el programa NVIVO8 ${ }^{7}$ que nos permitió, entre otras cosas, crear matrices longitudinales combinando tanto las categorías cualitativas entre sí, como las variables cualitativas con las cuantitativas.

Para abordar el fenómeno de las intenciones / decisiones residenciales como un proceso vimos necesario realizar los análisis desde varios enfoques y niveles. En los estudios cualitativos longitudinales existen dos enfoques principales: 1.) Enfoque transversal recurrente y 2.) Enfoque de trayectoria. Mientras que el primer enfoque permite analizar las experiencias /los hechos /los temas y los cambios en el tiempo a nivel del grupo, o la muestra completa de estudio, el segundo, se concentra en los cambios a nivel individual o en pequeños grupos de individuos (Grossoehme y Lipstein 2016). Dependiendo de la pregunta que queríamos contestar utilizamos uno u otro enfoque, o la combinación de ambos. A continuación, presentaremos algunos de los análisis llevados a cabo con el

\footnotetext{
${ }^{7}$ NVivo qualitative data analysis Software; QSR International Pty Ltd. v.8, 2008.
} 
fin de mostrar qué es lo que puede aportar un análisis cualitativo longitudinal al estudio del fenómeno de proyecto migratorio.

\subsection{Las dinámicas en la evolución de las intenciones/decisiones residenciales}

Con el fin de observar y describir la evolución de las intenciones residenciales utilizamos primero el enfoque transversal recurrente. Establecer la persistencia intencional para las intenciones a medio plazo ha sido una tarea compleja. Durante la evaluación se han tenido en cuenta las intenciones a corto y medio plazo expresados en el año 2009 y se han comparado con las intenciones tanto a corto (1 año) como medio plazo (5 años) expresados en el año 2012. Repetimos el mismo proceso de comparación con las intenciones expresadas en 2015. A la vez se observó el discurso sobre las fechas de las (no)acciones esperadas. Con respecto a la comparación de las intenciones a largo plazo, puede parecer extraño que se comparen intenciones para dos fechas distintas. Es decir, en realidad en el año 2009 se preguntaba sobre lo que iba ocurrir en el año 2024; y en el año 2012 se hacía referencia al año 2027; y en el año 2015 al año 2030. Sin embargo, según la experiencia del trabajo de campo cuando se interroga sobre un período tan amplio los entrevistados no suelen pensar en las fechas exactas, y la cuestión del horizonte es mucho más importante, y por ello la comparación sigue siendo factible.

Entonces, este tipo de análisis (véase la tabla 3) nos permitió evaluar el grado de la persistencia/estabilidad intencional en el tiempo. Luego, pudimos distinguir entre los casos que formaron su intención (desde la indecisión hacía una intención de retornar, quedarse o re-emigrar), los que cambiaron de intención (desde una intención definida hacia otra o hacia la indecisión), y los que mantuvieron la misma intención (sea de quedarse, retornar o no tener una intención aun definida).

Mientras que el enfoque cuantitativo longitudinal al estudio de las intenciones tiene como objetivo evaluar las (in)variaciones en el perfil sociodemográfico de los entrevistados según el tipo y la evolución de la intención en el tiempo; el enfoque cualitativo longitudinal se focaliza en la observación y comparación de los discursos en el tiempo y tiende a profundizar en las motivaciones y la lógica de los sucesos en el fenómeno. Nuestro análisis de variables cuantitativas evidenció que, por ejemplo, ni la edad de la primera emigración, ni el tiempo de residencia, ni la etapa de ciclo vital eran relevantes para la intención de retornar, re-emigrar y asentarse a lo largo plazo/permanentemente. No obstante, una mirada cualitativa a los datos cuantitativos sugirió que más que observar la etapa actual del ciclo vital en cada momento de observación era más revelador mirar su evolución durante todo el período de emigración y tener en cuenta su transición y la cantidad de eventos vitales experimentados. Es decir, era imprescindible realizar un análisis más dinámico al incorporar el enfoque mixto (enfoque transversal recurrente y a la vez enfoque de trayectoria). Para ello, dividimos la 
muestra según los 4 perfiles de itinerario intencional mencionados previamente ("permanente confirmado", "recién permanente", "indeciso", "cambiante"), los describimos según el tiempo biográfico (véase la tabla 4), y observamos las reflexiones y la evolución de discurso alrededor de estas variables en el tiempo según el perfil intencional. Más específicamente, observamos 3 dimensiones del tiempo biográfico: 1.) Etapas del ciclo vital (siguiendo la propuesta de Kley $\left.2011^{8}\right)$; 2.) Evolución de la experiencia profesional; 3.) Evolución de la vida familiar. De este modo, los discursos desvelaron que la proporción de cada dimensión y el tipo y diversidad de eventos vitales vividos en el país de destino y en al país de origen contribuyeron a la explicación de la construcción y/o redefinición de las intenciones residenciales. He aquí un ejemplo del itinerario intencional individual:

Natalia llegó a España en al año 2000, a los 28 años, por necesidad económica, tras haberse divorciado y dejado sus dos hijos con su exmarido en otro país de Europa del Este. Su proyecto migratorio ha estado marcado por una persistencia intencional de retornar y una fuerte convicción sobre su futura realización incluso después de haber traído su hija a España en el año 2008. Aunque la intención de retornar ha sido modificada en términos de temporalidad y el retorno ha sido aplazado, incluso varias veces a lo largo del proyecto migratorio, la vocación de una permanencia muy temporal ha marcado significativamente el curso de la vida laboral de Natalia en España. Durante los primeros 12 años en España, Natalia acotó sus posibilidades de crecer profesionalmente en España, ya que siempre había preferido trabajos menos cualificados o de bajo estatus laboral, pero mejor remunerados que los puestos que le fuesen más próximos profesionalmente y físicamente menos duros. A pesar de que había sufrido una desprofesionalización (en Ucrania trabajó como maestra de lengua y literatura ucraniana y en España pasó por empleos en la agricultura, el cuidado de los niños, y la limpieza), intentaba adquirir otro tipo de conocimiento (estudiando un curso de auxiliar de enfermería), para poder añadirle un mayor sentido a esta época de emigración, esperando poder aprovechar su experiencia en el extranjero para crecer profesionalmente en Ucrania, y pretendía llevar a cabo el tipo de regreso llamado 'retorno de innovación'9 . Además, la noción de retorno a medio plazo se reflejó también en ciertas estrategias relacionales. Natalia, consciente de que su estancia siempre había sido algo muy temporal, no hizo mucho esfuerzo ni puso mucho interés en crear un entorno relacional amplio y afectivo en España, más allá de lo necesario, lo cual supuso un obstáculo para poder sentirse que formaba parte plenamente de la sociedad de acogida: "Yo tengo mi situación diferente que los otros ucranianos. No tengo muchos amigos aquí. Si

${ }^{8}$ Motivándose en la investigación de Kley (2011), pero introduciendo algunos cambios en función de las particularidades del estudio y en función de nuestra muestra, la primera fase denominada 'edad adulta temprana' comprende aquellos individuos de entre 18 y 29 años de edad y sin hijos. La segunda, la 'fase familiar' contiene individuos de edad entre 18 y 60 años y con hijo(s) menor(es) de 10 años, y la tercera fase ('consolidación') incluye personas de la franja de edad de entre 30 y 60 años, sin hijos a cargo o con hijos mayores de 10 años.

${ }^{9}$ Un tipo de retorno que proviene de la tipología de retornados elaborada por Cerase (1974). 
te vienes aquí para trabajar es otra cosa. Sabes, si te vienes a trabajar no te da tiempo para muchas cosas." (primera oleada en 2009, tras 9 años de residencia en España) Este ejemplo es ilustrativo de la existente dualidad entre la agencia del inmigrante y su entorno relacional.

En 2012, Natalia y su hija fijaron una fecha de regreso y comenzaron a prepararse. Poco antes de los movimientos preeuropeos de Euromaidán a finales de 2013, Natalia y su hija estaban listas para regresar, pero cuando vieron la situación en Ucrania cambiaron de opinión y se quedaron en España. En la entrevista de la tercera oleada en 2015, su intención de regresar había cambiado hacia la indecisión y una apertura hacia un posible asentamiento a largo plazo. Cabe precisar que en el período entre la segunda y la tercera oleada, pasaron varios acontecimientos: Natalia terminó su formación y empezó trabajar en una residencia como enfermera; dejó de planificar el retorno; encontró una pareja española y empezó integrarse más en la sociedad española; su hija ha tenido un niño lo cual le llevó a asumir el rol de abuela. Estos acontecimientos vinculados a las tres dimensiones del tiempo biográfico y concentrados en un período de tiempo relativamente corto contribuyeron al cambio del discurso de Natalia. Allí, se apreció la importancia del factor del 'tiempo bien aprovecho', y si se le añade el factor de la edad, la opción de retorno sería para Natalia, como 'empezar desde cero'.

Este ejemplo muestra como la entrevistada objetiviza el tiempo para la planificación la cual luego se subjetiviza en la formación / cambio de las intenciones y decisiones según el uso que se le da al tiempo. Este tiempo finalmente se relativiza y adquiere un sentido propio, ayudando a definir presente y futuro del individuo. 


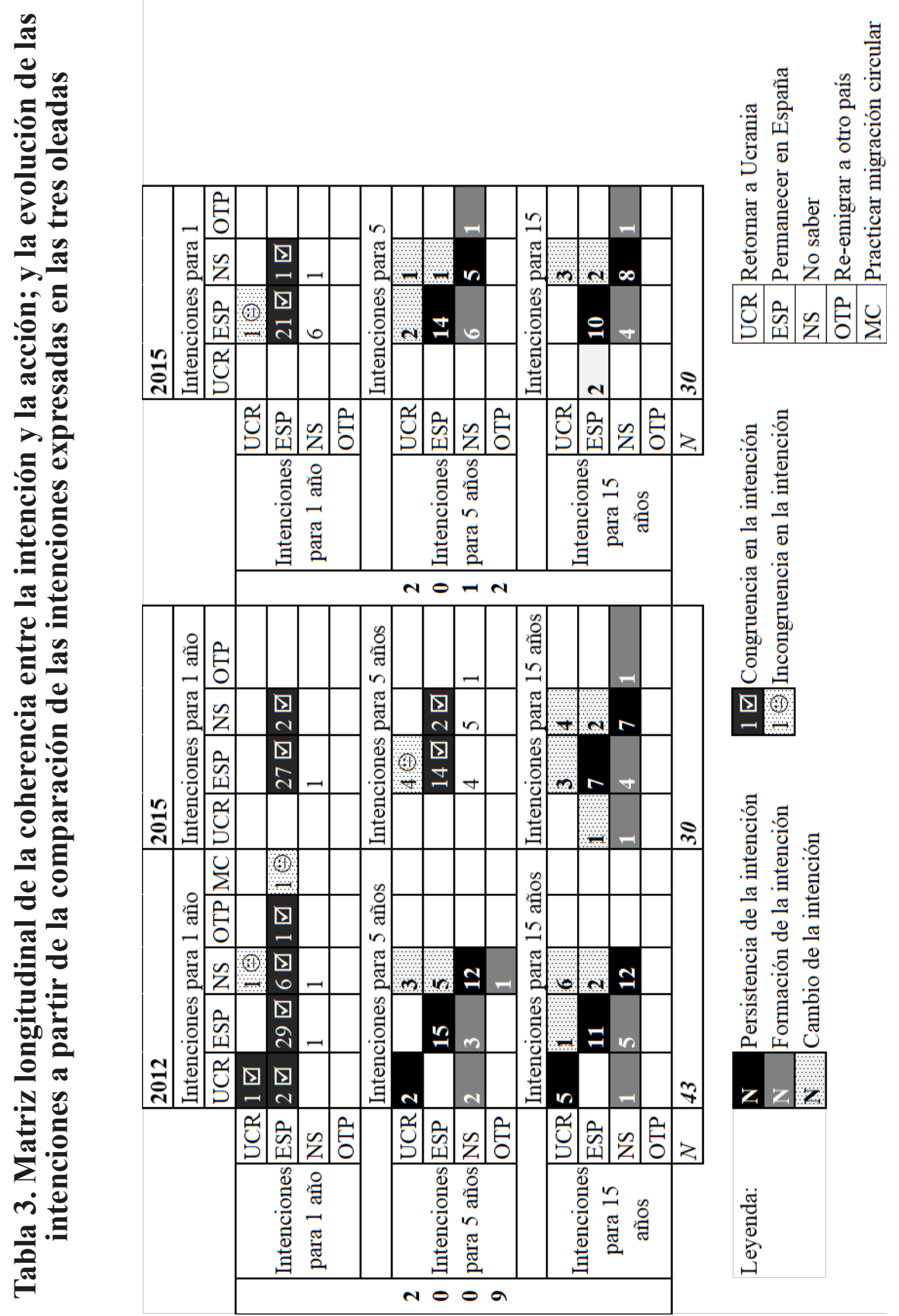




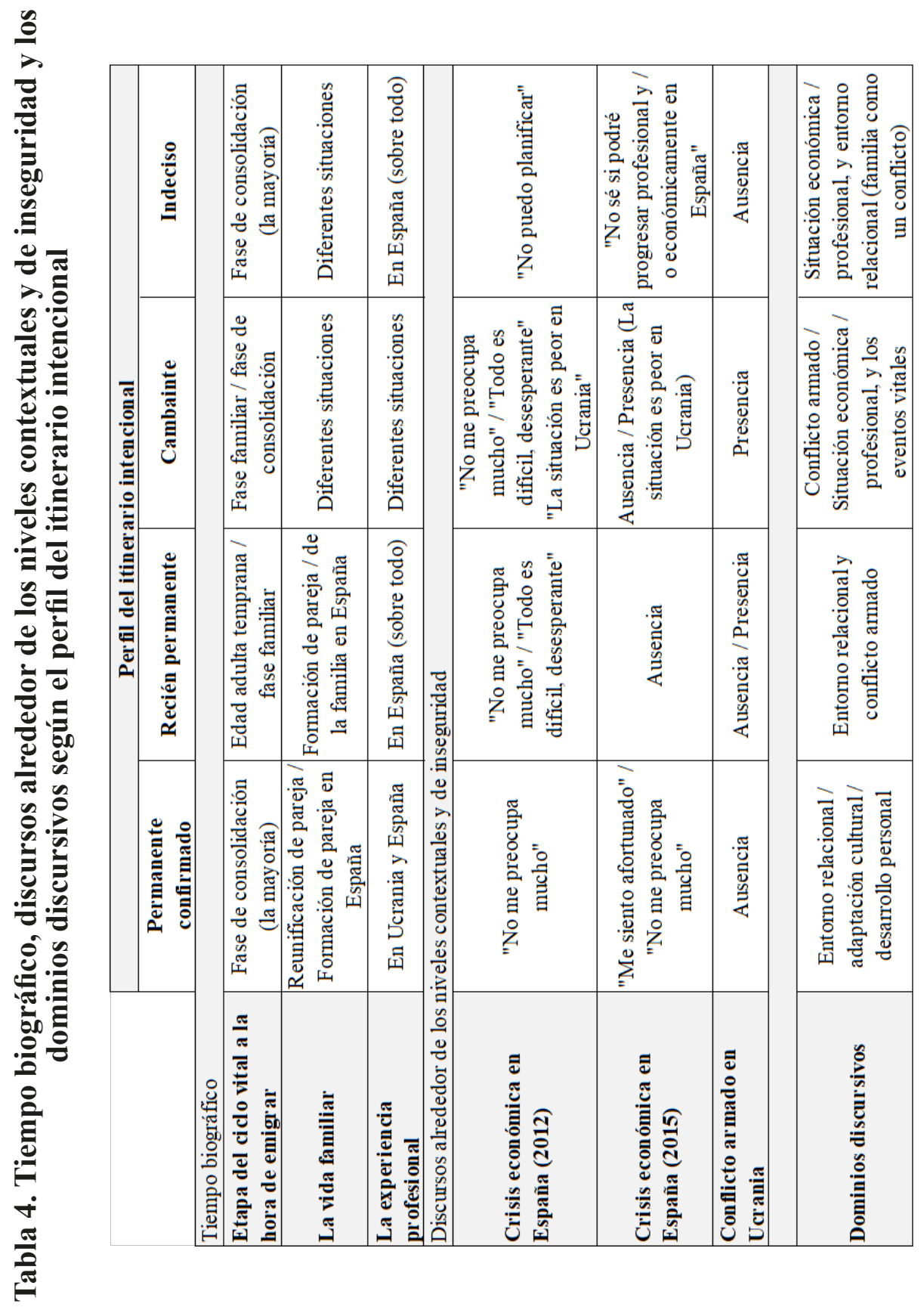


La tabla 4 es un resumen simplificado de los resultados del proceso analítico, permitiéndonos describir los perfiles de itinerarios intencionales. Vemos, por ejemplo, que aquellos que habían formado su intención de permanecer en España durante los 6 años de observación (grupo de los "recién permanentes"), llegaron a España en la fase de la edad adulta temprana o en la fase familiar y son los que habían experimentado más eventos vitales en España de todos los perfiles intencionales. Concretamente, estas personas compartieron la experiencia de varios de los siguientes eventos vitales en España: formación de pareja, nacimiento del primer hijo/a, escolarización del hijo/a, primera experiencia laboral, crecimiento profesional, y entonces, la frase más pronunciado entre estas personas ha sido "Yo he hecho mi vida aquî". Por otro lado, si comparamos el grupo de los "recién permanentes" con los "cambiantes" que redefinieron su intención residencial hacía otra dirección, vemos que éstos también habían vivido varios eventos / cambios vitales en España, pero tratándose más bien de eventos negativos (separación, divorcio, muerte de un miembro de la familia). Sobre todo, se trató de cambios recientes a los cuales los entrevistados aún no tuvieron tiempo adaptarse.

A continuación, analizamos las similitudes / diferencias y particularidades en los discursos en relación con los factores macroestructurales (crisis económica y guerra) en distintas oleadas y según el perfil de itinerario intencional. Además, buscamos a identificar los dominios discursivos propios de cada perfil intencional para así: 1.) Evaluar el peso "real" de los elementos propuestos y observados, pero contextualizados en un paisaje argumental complejo; 2.) Dejar espacio para que se visualicen otras posibles influencias en las intenciones y decisiones residenciales. De esta forma, fuimos capaces de comprender mejor porqué los entrevistados en cada perfil intencional vivieron y enfrentaron la incertidumbre económica y geopolítica, y sus efectos de diferente manera; y porqué estos factores jugaron el rol en el cambio de las intenciones / decisiones de unos y no de otros.

Así, pudimos apreciar (véase en la tabla 4) que la guerra en Ucrania no afectó las motivaciones intencionales de los "permanentes confirmados" ni de los "indecisos". Además, vimos que muy pocos entrevistados cambiaron de intención principalmente por la aparición y/o perduración del conflicto armado en Ucrania. Por el contrario, la mayor parte de los entrevistados había sido afectada negativamente a nivel económico, ya que perdieron su trabajo al menos una vez durante los 6 años de observación. Sin embargo, los efectos de la crisis económica no se reflejaron en las intenciones de todos los perfiles intencionales. Si bien para los "indecisos" la crisis económica fue la principal fuente de indecisión residencial, ésta nunca figuró como el único factor. Mientras que en 2012, la mayoría de los entrevistados insistieron en que la situación de inseguridad laboral en España no les permitía planificar su futuro; en 2015, la mayor preocupación de estos mismos entrevistados fue: "No sé si podré progresar profesional y /o económicamente en España". En comparación con el grupo de los "permanentes confirmados" que no consideraron la crisis económica como algo por lo que cambiar las intenciones, gracias a que se sentían apoyados por sus redes, los "indecisos" le daban mucha importancia a la vida profesional. Se habían propuesto 
metas profesionales que querían alcanzar, todavía querían evolucionar profesionalmente, pero no estaban seguros de poder lograrlo en España. Sin embargo, esta incertidumbre no los llevó a formar la intención de regresar, ya que la mayoría tenía más experiencia laboral en España que en Ucrania (algunos de ellos nunca habían trabajado en Ucrania porque habían salido de Ucrania en la fase adulta temprana). Su situación laboral representaba para ellos tanto un factor de atracción como un factor de expulsión, ya que dudaban entre los sentimientos de arraigo profesional en España y la precariedad laboral. Asimismo, entre los dominios discursivos para este perfil intencional destacaba la familia como una fuente de conflicto. Siempre se trataba de un conflicto entre la obligación familiar y las expectativas personales, o profesionales. A menudo, había desacuerdo entre los miembros de la familia sobre si permanecer o irse de España.

De esta manera comparamos todos los perfiles de itinerarios intencionales entre sí, centrándose sobre todo en la explicación de cómo se forma la intención de asentarse en el país de destino a largo plazo o permanentemente. Este análisis comparativo fue diseñado con el fin de enfrentarse a las limitaciones de nuestra muestra (casos perdidos, pocos casos con intenciones de retornar, etc.). Si bien la pérdida de casos a lo largo de un estudio longitudinal es un fenómeno normal y esperado, no se puede obviar que esto implica algunos riesgos analíticos y que se debe tener cuidado al interpretar los resultados. Tener información sobre casos perdidos (debido al retorno / re-emigración a otro país / falta de contacto y / o renuencia a continuar en el estudio longitudinal) sin duda hubiera ayudado a comprender mejor la complejidad de la interacción de elementos en el proceso intencional / decisional. Sin embargo, cabe mencionar que nuestra muestra contiene también migrantes que, aunque regresaron a Ucrania y volvieron a emigrar a España, participaron en todas las oleadas de la encuesta. El seguimiento de estos casos en particular ayudó a arrojar luz sobre los elementos que influyen en la intención / decisión de no permanecer en el país de origen.

Por lo tanto, la mayor contribución de la aproximación longitudinal cualitativa fue que se consiguió mostrar la existencia de una gran diversidad de líneas argumentales y observar los procesos causales cognitivos, lo que posibilitó una mayor profundización sobre cómo se modelan las intenciones. Luego, se demostró la importancia de las vivencias, las expectativas y las esperanzas en el proceso de formación de las intenciones residenciales. De este modo, se identificaron los factores y circunstancias, que influyen en cómo el individuo percibe y considera como importante las condiciones macro-estructurales en el país de origen y en el país de destino. Al fin y al cabo, el hecho de que haya crisis económica no es tan determinante para las intenciones, como la manera en la que uno la vive que, a su vez, está determinada por la situación personal y relacional del individuo. 


\subsection{Las dinámicas relacionales en el proceso intencional / decisional}

Dado que el análisis de los dominios discursivos subrayó la importancia del entorno relacional en el proceso de intenciones / decisiones residenciales insistimos en profundizar sobre este aspecto centrándose en el enfoque de trayectoria. El enfoque transversal recurrente a veces no permite visualizar algunos factores de cambio en el tiempo dado que se evalúa la sub/muestra o población como un conjunto (Calman et al. 2013). Entonces, analizamos los trece casos, mapeando la evolución de la composición y la estructura de la red personal de cada individuo entre la primera y cuarta oleada, y la vinculamos con el proceso del proyecto migratorio (teniendo en cuenta tanto los itinerarios intencionales como los discursos alrededor de los factores micro y macro-estructurales expresados en todas las oleadas). A continuación, presentaremos un caso para mostrar el potencial de este análisis ${ }^{10}$.

\section{Figura 2. Caso de Yuriy. Evolución de su red personal y de la intención a largo plazo entre los años 2009 y 2016}

\section{9, Intención de retornar a Ucrania}

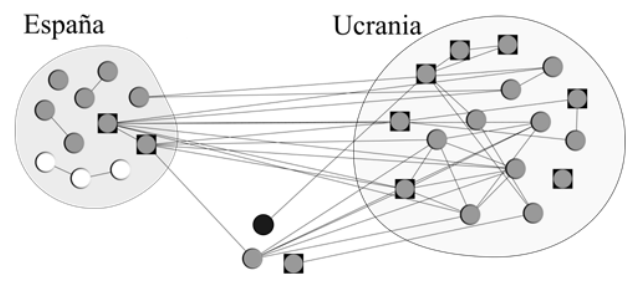

2016, Indeciso

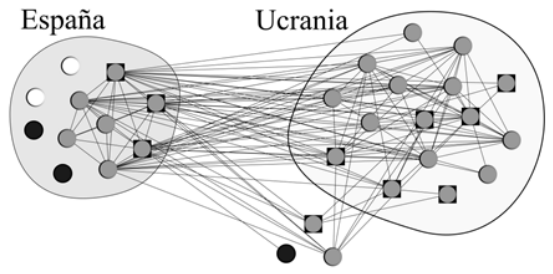

Leyenda: QUcranianos CEspañoles $\bigcirc$ Otras nacionalidades DPresencia en la oleada 1 y 2

Caso de Yuriy (véase la figura 2) es un hombre que llegó por la primera vez a España a la edad de 25 años (en 2007) y que a lo largo de su proyecto migratorio experimentó varios cambios: personal (se casó y tuvo 2 hijos), profesional (pasó por una reconversión profesional) y geográfico (en 2013 regresó a Ucrania por la crisis en España, luego en 2014 volvió a emigrar a España por la guerra y la situación socio-económica en Ucrania, por la cual se vio incapaz de comenzar su negocio y mantener su familia). Aunque parezca que la red de

10 Hay que subrayar que aquí presentamos solo una forma de analizar las redes personales longitudinales y algunos de los resultados significativos. En realidad, el análisis de redes personales longitudinales (cuali-cuantitativo) tiene un enorme potencial, se pueden tener en cuenta muchas de las variables de estructura y de composición. Además, se pueden elaborar distintos modelos evolutivos (véase p. ej. en Lubbers et al. 2010) que pueden ser contrastados con otras variables cualitativas. Sin embargo, esto sobrepasaría los objetivos del presente texto. 
Yuriy no cambió entre los años 2009 y 2016, el discurso reveló que tras el retorno y la re-emigración, la red tuvo que ser reconstruida nuevamente en al año 2014, para alcanzar la misma composición que en el año 2009. Sin embargo, a nivel de la estructura se produjeron cambios más significantes. La conectividad transnacional entre sus contactos ucranianos aumentó dramáticamente. Esto está vinculado a la transición de un entorno compuesto principalmente de amistades a un entorno más orientado a la familia. La presencia de muchos miembros de la familia tanto en el país de origen como en el país de acogida permitió esta fuerte conectividad transnacional. Además, Yuriy explicó que la prevalencia del mundo ucraniano local y extra-local en su entorno relacional, y la idea de mudarse a una ciudad española más pequeña no permitieron una mejor integración relacional en el mundo español. Por lo tanto, aunque el entrevistado en 2016 rechazó el retorno a Ucrania, no formó la intención de permanecer en España ni a medio ni a largo plazo.

Este tipo de análisis visual y cualitativo complejo que considera varios niveles de influencia permitió identificar algunas variables que en otros análisis parecían no cobrar mucha importancia, pero aquí ganaron en fuerza explicativa. En efecto, al prestar especial atención al fenómeno circulatorio entre origen y destino (visitas o/y retorno/re-emigración), el análisis indicó que la secuencia de causa y efecto de los acontecimientos durante el proceso de formación de la intención de asentarse en el país de destino, está influenciado por el género. Mientras que en el caso de las mujeres, el proceso de integración relacional está más marcado por los micro-factores (cambios en el ciclo vital, pareja española, hijos, matrimonio), en el caso de los hombres, son más los factores macro-estructurales, contextuales. Específicamente, las intenciones / decisiones residenciales de los hombres están altamente determinadas por el riesgo de ser reclutados por el ejército ucraniano. Como resultado, la mayoría de los hombres (o esposos de mujeres) entrevistadas no visitaron Ucrania entre 2014 y 2015, lo cual en muchos casos llevó a la reducción de los contactos en el origen. En consecuencia, esto impactó su grado de arraigo relacional y sentimientos de pertenencia al país de origen, y entonces, afectó negativamente las intenciones de retornar.

Otra contribución del enfoque cualitativo de trayectoria al estudio del proyecto migratorio fue que permitió ver que el tipo de intención (o itinerario intencional) y la (no)vocación de la temporalidad se reflejaban en las estrategias formativo-laborales, legales y transnacionales (visitas y comunicación al/con el origen, reunificación familiar) del inmigrante durante su proyecto migratorio. Por un lado, esto posibilitó la diferenciación entre las prácticas estratégicas llevadas a cabo por los entrevistados y orientadas hacia el futuro lugar de residencia de las prácticas que no estaban motivadas por este fin. Por otro lado, aunque descifrar el proceso causal entre la formación de la intención/ decisión residencial, el entorno relacional y otros factores micro y macro estructural es una tarea muy complicada, el enfoque longitudinal que tiene en cuenta los fines de las prácticas sin duda ayuda a arrojar la luz sobre el proceso causal. Así fue, por ejemplo, el caso de la variable de la reunificación familiar que de modo general en los análisis de las intenciones residenciales se trata como un hecho 
(haber reunificado o no), pero se suele dejar de lado todo el comportamiento del inmigrante alrededor de esta práctica (ej. los intentos fallidos, la planificación y la acción llevada a cabo para conseguir reunificar un/os familiar/es, las motivaciones). Los análisis mostraron que en el caso de los ucranianos la reunificación familiar casi siempre causó la prolongación de la estancia en España, pero nunca fue precedida de la formación de la intención de permanecer en España a largo.

\section{CONCLUSIONES}

El presente artículo ofrece una reflexión metodológica en torno al modo de cómo abordar las intenciones / decisiones residenciales en la migración internacional, y así comprender la formación, modificación y consolidación de las intenciones y decisiones de retorno, asentamiento y re-emigración. Se defiende el estudio de este fenómeno como un proceso dinámico, contemplándolo como algo continuo, resultado de una negociación constante entre el actor y su entorno. A partir del estudio de caso de los ucranianos en Madrid (que articula las dimensiones temporales, espaciales y relacionales a la vez) este artículo muestra las posibilidades, las aportaciones y los desafíos de la aproximación longitudinal cualitativa.

Se pueden subrayar varias ventajas que tiene el enfoque longitudinal cualitativo frente otros enfoques metodológicos para el estudio de intenciones / decisiones migratorias.

Si comparamos el enfoque longitudinal cualitativo con otros enfoques cualitativos que se basan en entrevistas retrospectivas, la virtud del primero consiste en que se minimizan las distorsiones del recuerdo selectivo habitualmente observado en estudios donde se reconstruye el pasado a partir del presente (BotíaMorillas y Jurado-Guerrero 2018: 30). Aunque se puedan utilizar técnicas de recogida de datos que pueden ayudan a limitar dicho sesgo, por ejemplo, apoyándose en los eventos vitales u otros importantes (preguntando si la intención residencial cambió después del nacimiento del hijo, o después de la pérdida del trabajo, o si coincidió con del estallido de la crisis, etc.), esto no garantiza la reducción del sesgo. De hecho, la experiencia de un trabajo de campo complementario de este largo estudio que se condujo en Ucrania con retornados en 2010 (con una muestra aparte cuyo análisis no forma parte del presente artículo) y donde se emplearon solo entrevistas retrospectivas, mostró que si bien situar las acciones (el retorno llevado a cabo) en el tiempo es relativamente fácil, reconstruir la formación y/o modificación de intenciones y poner fechas al suceso de toma de decisiones ya no lo es tanto. Además, si añadimos el nivel relacional al estudio, obtener una foto fija fiel de la composición y la estructura de redes personales desde el enfoque retrospectivo y ponerla en relación con la evolución del proyecto migratorio es una tarea aún más ardua, por no decir imposible.

De aquí se despliega otra virtud del enfoque longitudinal cualitativo frente las entrevistas retrospectivas, y es que éste es más apto para los estudios donde se examina la intersección de más de dos niveles. Recordemos que en el estudio 
UCRAM 2009-2016 se ha examinado la intersección de tres niveles - nivel micro (individual - las intenciones, las motivaciones, el curso de vida) - nivel meso (relacional - imbricación en las redes personales) y nivel macro (contextual diferentes niveles de inseguridad). El presente estudio demuestra que recoger la información y evaluar el grado de influencia del impacto de eventos vitales, y la vez, el de los factores macroestructurales en las intenciones/decisiones residenciales es más riguroso desde un enfoque longitudinal donde las entrevistas repetidas de los entrevistados se sitúan en los mismos períodos de observación que en el presente estudio están definidos según los cambios contextuales en los países de destino y de origen. Así las respuestas de todos los sujetos a los cambios macroestructurales están recogidas en el momento sin tener que reconstruir retrospectivamente varios ejes de la temporalidad lo que sin duda requería mucho más esfuerzo tanto para el entrevistado (en cuanto a la memoria) como para el entrevistador (en cuanto a las estrategias de preguntar).

$\mathrm{Si}$ ahora comparamos el enfoque longitudinal cualitativo con el enfoque longitudinal cuantitativo para el estudio de las intenciones / decisiones migratorias, podemos enumerar varias ventajas que priman del primero sobre el segundo.

Por un lado, el primero es capaz de identificar mejor los cambios residenciales bien reales o potenciales que ocurren entre los períodos de observación, (p.e. retorno y pronta re-emigración; modificaciones en las intenciones). Esto se debe a que las entrevistas cualitativas dejan espacio para una mayor profundización en las trayectorias, mientras que los cuestionarios que en su mayoría contienen preguntas cerradas pueden enmascarar la movilidad ocurrida fuera de los momentos de colección de datos. Igualmente, las entrevistas en profundidad sobre la composición y estructura de las redes personales ayudan a aclarar los (no)cambios en sus configuraciones (aquí podemos recordar el ejemplo de Yuriy cuya red sufrió muchos cambios entre los períodos de observación, sin embargo, la comparación de la composición de las redes entre 2009 y 2016 indicaba más bien una estagnación).

Por otro lado, el enfoque longitudinal cualitativo parece ser más adecuado para descifrar y comprender los procesos causales. Entre otras cosas, porque el investigador no está obligado a escoger el ángulo desde el que va a estudiar el sujeto. Hay que señalar que en la gran mayoría de estudios cuantitativos (sean de tipo transversal o sean de tipo longitudinal) la intención/decisión, normalmente, se trata como 'variable explicativa' y raramente como una 'variable dependiente'. Sin duda, distinguir entre qué es causa y qué es efecto, no siempre es una tarea fácil, simple y posible. Sin embargo, el presente estudio entra en discusión acerca de la posible bidireccionalidad de causas y efectos del proceso. De este modo, los análisis han mostrado que la comparación de redes estáticas correspondientes a dos períodos de tiempo diferentes y a la vez el análisis de la evolución del proyecto migratorio y de los discursos alrededor de él sin duda ayuda a comprender mejor sus interrelaciones y la lógica de los sucesos en el fenómeno.

Por último, el artículo muestra bien la capacidad del enfoque cualitativo longitudinal para abordar las intenciones / decisiones como un proceso dinámico que ayuda captar cómo los sujetos y sus vivencias, expectativas y esperanzas 
evolucionan a lo largo del tiempo. Gracias a este enfoque el investigador puede comprender mejor cómo los migrantes utilizan el tiempo, cómo se adaptan a circunstancias cambiantes entre el país de origen y el de destino, y cuál es el grado de la vocación de la temporalidad en cuanto a sus futuros planes la cual luego se refleja en las estrategias formativo-laborales, legales y transnacionales (visitas y comunicación al/con el origen, reunificación familiar) durante su proyecto migratorio.

Ahora bien, ningún enfoque es perfecto y el enfoque longitudinal cualitativo también tiene sus limitaciones. Entre los principales y que se extienden al estudio de cualquier fenómeno, no solamente al de las intenciones/decisiones migratorias son: 1) es costoso en tiempo y en esfuerzo, tanto para la recogida y la organización de datos como para sus análisis; 2) conlleva el problema de 'adelgazamiento' de la muestra. Por un lado, el investigador debe prestar atención especial a la hora de construir la muestra inicial, y por el otro lado, se debe tener mucho cuidado a la hora de interpretar los datos. A veces la 'mortalidad' de ciertos perfiles en la muestra no permite al investigador a responder a todas las preguntas o le obliga a redefinir algunas de ellas o reorientar los objetivos (por ejemplo, en el caso de estudios sobre migrantes, hay que tener en cuenta que la probabilidad de encontrar y re-entrevistar a un retornado puede ser menor que a un inmigrante que no se haya mudado ni su situación personal/familiar haya cambiado).

Como punto final, hay que recopilar los elementos que han sido discutidos a lo largo del texto y que requieren una mayor reflexión a la hora de planificar y llevar a cabo un estudio con el enfoque longitudinal cualitativo. A parte del diseño de la muestra adecuado, hay que premeditar de cómo espaciar las oleadas en función de los ejes temporales analizadas, pero siempre teniendo en cuenta que al trabajar con una población 'móvil' no hay que dejar pasar demasiado tiempo entre los períodos y emplear ciertas estrategias de seguimiento de los entrevistados. Otra atención especial requiere la conceptualización del proyecto migratorio y la adecuada formulación de las preguntas. Las palabras utilizadas y la (no)delimitación temporal del proyecto migratorio va a influir en las respuestas, por lo que es muy importante reproducir las misma preguntas claves en todas las oleadas. Luego, huelga decir que todos los estudios de tipo longitudinal requieren un esfuerzo importante en la gestión de la información recogida para preservar la confidencialidad y el anonimato de datos personales. Por último, se ofrecen varias aproximaciones analíticas a los datos cualitativos longitudinales. El presente artículo ha mostrado que la incorporación del enfoque mixto - el de transversal recurrente (que se centra en experiencias/los hechos y los cambios en el tiempo a nivel del grupo) con el de la trayectoria (que se focaliza en los cambios a nivel individual) - es muy fructífera para abordar y comprender las dinámicas del fenómeno intencional / decisional en migraciones. 


\section{REFERENCIAS BIBLIOGRÁFICAS}

BOTÍA-MORILLAS, C., Y JURADO-GUERRERO, T. (2018): "El proceso de una investigación cualitativa longitudinal sobre la transición a la maternidad y paternidad en España”, EMPIRIA. Revista de Metodología de Ciencias Sociales, 41 septiembre-diciembre, pp. 33-56.

BOYD, M. (1989): "Family and Personal Networks in International Migration: Recent Developments and New Agendas", International Migration Review, 23, 3, pp. 638669.

BROWN, D. L. (2002): "Migration and Community: Social Networks in a Multilevel World", Rural Sociology, 67, 1, pp. 1-23.

CAÏS, J., FOLGUERA, L., Y FORMOSO, C. (2014): "Investigación Cualitativa Longitudinal", Cuadernos Metodológicos 52, Madrid: CIS, pp. 148.

CALMAN, L., BRUNTON, L. Y MOLASSIOTIS, A. (2013): "Developing longitudinal qualitative designs: lessons learned and recommendations for health services research", BMC Medical Research Methodology, 13:14, pp. 1-10.

CERASE, F. P. (1974): "Expectations and reality: a case study of return migration from the United States to Southern Italy", International Migration Review, 8, 2, pp. 245262.

CHAVEZ, L. R. (1994): "The Power of the Imagined Community: The Settlement of Undocumented Mexicans and Central Americans in the United States", American Anthropologist, 96, 1, pp. 52-73.

COULTER, R., VAN HAM, M., Y FEIJTEN, P. (2010): “A Longitudinal Analysis of Moving Desires, Expectations and Actual Moving Behaviour", en IZA Discussion Papers 5277, Institute for the Study of Labor (IZA) (Ed.). Bonn, pp. 1-23.

DURAND, J. (2004): "Ensayo teórico sobre la migración de retorno. El principio del rendimiento decreciente", Cuadernos Geográficos, 35, pp. 103-116.

FARRALL, S. (2006): "What is qualitative longitudinal research?", en LSE Methodology Institute Discussion Papers - Qualitative Series, Paper 11, London: London School of Economics. Disponible en http://www2.lse.ac.uk/methodology/pdf/QualPapers/Stephen-FarrallQual\%20Longitudinal\%20Res.pdf [consulta: 10-9-2018].

FARRALL S., HUNTER B., SHARPE G., Y CALVERLEY, A. (2016): "What 'works' when retracing sample members in a qualitative longitudinal study?", International Journal of Social Research Methodology, 19, 3, pp. 287-300.

FAWCETT, J. T. (1989): "Networks, linkages, and migration systems", International Migration Review, 23, 3, pp. 671-680.

FLIEGENSCHNEE, K. (2006): "Children should be a part of my life, but I don't know how to manage it. A qualitative fertility study of highly educated women in Vienna", European Population Conference, 21-24, Liverpool, UK.

GMELCH, G. (1980): "Return Migration”, Annual Review of Anthropology, 9, pp. 135159.

GURAK, D. T., Y CASES, F. (1992): "Migration Networks and the Shaping of Migration Systems", en M. M. Kritz, et al. (dir.), International Migration Systems: A Global Approach, Oxford: Clarendon Press, pp. 150-176.

DE JONG, G.F. y GARDNER, R.W. (1981): "Migration Decision Making: Multidisciplinary Approaches to Microlevel Studies in Developed and Developing Countries", New York: Pergamon, 394 p. 
GROSSOEHME, D., Y LIPSTEIN, E. (2016): “Analyzing longitudinal qualitative data: the application of trajectory and recurrent cross-sectional approaches", BMC Research Notes, 9: 136, pp.1-5.

GÜNGÖR, N. D., Y TANSEL, A. (2010): "Brain Drain from Turkey: Return Intentions of Skilled Migrants", Working Papers 2010/4. En Turkish Economic Association (Ed.). Ankara.

HOANG L.A. (2011): "Gender Identity and Agency in Migration Decision-Making: Evidence from Vietnam", Journal of Ethnic and Migration Studies, 37:9, pp. 14411457.

HOLLAND J. (2007): “Qualitative Longitudinal Research: Exploring ways of researching lives through time", Real Life Methods Node of the ESRC National Centre for Research Methods Workshop held at London South Bank University. Disponible en http://www.reallifemethods.ac.uk/training/workshops/qual-long/documents/qlworkshop-holland.pdf. [consulta: 15-9-2018].

HOSNEDLOVÁ, R. (2014): "De la intención de retornar al retorno. El papel de las redes en la experiencia de los ucranianos en Madrid”, Tesis doctoral, Madrid, Universidad Complutense de Madrid.

KHOO, S.E. (2003): "Sponsorship of Relatives for Migration and Immigrant Settlement Intention", International Migration, 41, 5, pp. 177-199.

KLEY, S. (2011): "Explaining the Stages of Migration within a Life-course Framework", European Sociological Review, 27, 4, pp. 469-486.

KODZI, I. A., JOHNSON, D. R., Y CASTERLINE, J. B. (2010): "Examining the predictive values of fertility intentions among Ghanaian women", Demographic Research, 22, 30, pp. 965-984.

LUBBERS, M.J., MOLINA, J.L., LERNER, J., BRANDES, U., ÁVILA, J., MCCARTY, C. (2010): 'Longitudinal analysis of personal networks. The case of Argentinean migrants in Spain", Social Networks, 32, pp. 91-104.

MCCARTY, C., Y KILLWORTH, P. (2007): "Impact of methods for reducing respondent burden on personal network structural measures", Social Networks, 29, pp. 300315.

MCHUGH, K. E. (1984): "Explaining migration intentions and destination selection", The Professional Geographer, 36, 3, pp. 315-325.

MITCHELL, D., Y GRAY, E. (2007): "Declining fertility: Intentions, attitudes and aspirations", Journal of Sociology, 43, 1, pp. 23-44.

PIRES, A. P. (1997) : "Échantillonnage et recherche qualitative : essai théorique et méthodologique" en La recherche qualitative: enjeux épistémologiques et méthodologique, Montréal, Gaëtan Morin éditeur, pp. 113-167.

PORTES, A., Y SENSENBRENNER, J. (1993): "Embeddedness and Immigration: Notes on the Social Determinants of Economic Action", The American Journal of Sociology, 98, 6, pp. 1320-1350.

SALDANA, J. (2003): "Longitudinal qualitative research: analyzing change through time", Walnut Creek, California: AltaMira Press, pp. 199.

SEIDMAN, I. E. (1998): "Interviewing as qualitative research: A guide for researchers in education and the social sciences" (2nd ed.) New York: Teachers College Press.

SELL, R. R., Y DE JONG, G. F. (1983): "Deciding Whether to Move: Determinants of Alternative Paths Within the Migration Decision Process", Sociology and Social Research, 67, pp. 146-165. 
STAMM, S. (2006): "Social Networks Among Return Migrants to Post-War Lebanon", en CIS Working Papers, 9: UNI ETH Zurich: Center for Comparative and International Studies (CIS).

THOMSON, R. (2007): "The Qualitative Longitudinal Case History: Practical, Methodological and Ethical Refections", Social Policy and Society, 6, .pp 571-582.

TSUDA, T. (1999): “The permanence of 'Temporary' Migration: The 'Structural Embeddedness' of Japanese-Brazilian Immigrant Workers in Japan", The Journal of Asian Studies, 58, 3, pp. 687-722.

VAN DALEN, H. P., GROENEWOLD, G., Y SCHOORL, J. J. (2005): “Out of Africa: What drives the pressure to emigrate?", Journal of Population Economics, 18, 4, pp. 741-778.

WINIARSKA, A. (2017): "Qualitative Longitudinal Research: Application, Potentials And Challenges", en The Context Of Migration Research. CMR Working Papers 103/161. P. 29.

https://depot.ceon.pl/bitstream/handle/123456789/15189/WP103161.pdf?sequence=1, [consulta: 10-10-2018].

WRAY N, MARKOVIC M, y MANDERSON L. (2007): "Researcher Saturation: The Impact of Data Triangulation and Intensive-Research Practices on the Researcher and Qualitative Research Process". Qualitative Health Research, 17, 10, pp. 13921402.

YATES L. (2003): "Interpretative claims and methodological warrant in small-number, qualitative, longitudinal research", International Journal of Social Research Methodology, 6, 3, pp. 223-232. 
